University of Nebraska - Lincoln

DigitalCommons@University of Nebraska - Lincoln

4-6-2012

\title{
Mechanical Writing of Ferroelectric Polarization
}

\author{
Haidong Lu \\ University of Nebraska-Lincoln, s-hlu4@unl.edu \\ C.-W. Bark \\ University of Wisconsin-Madison \\ D. Esque de los Ojos \\ Universitat Politecnica de Catalunya, Barcelona, Spain \\ J. Alcala \\ Universitat Politecnica de Catalunya, Barcelona, Spain \\ Chang-Beom Eom \\ University of Wisconsin-Madison, eom@engr.wisc.edu \\ See next page for additional authors
}

Follow this and additional works at: https://digitalcommons.unl.edu/physicsgruverman

Part of the Physics Commons

Lu, Haidong; Bark, C.-W.; Esque de los Ojos, D.; Alcala, J.; Eom, Chang-Beom; Catalan, G.; and Gruverman, Alexei, "Mechanical Writing of Ferroelectric Polarization" (2012). Alexei Gruverman Publications. 56. https://digitalcommons.unl.edu/physicsgruverman/56

This Article is brought to you for free and open access by the Research Papers in Physics and Astronomy at DigitalCommons@University of Nebraska - Lincoln. It has been accepted for inclusion in Alexei Gruverman Publications by an authorized administrator of DigitalCommons@University of Nebraska - Lincoln. 


\section{Authors}

Haidong Lu, C.-W. Bark, D. Esque de los Ojos, J. Alcala, Chang-Beom Eom, G. Catalan, and Alexei Gruverman 


\title{
Mechanical Writing of Ferroelectric Polarization
}

\author{
H. Lu, ${ }^{1}$ C.-W. Bark, ${ }^{2}$ D. Esque de los Ojos, ${ }^{3}$ J. Alcala, ${ }^{4}$ C. B. Eom, ${ }^{2}$ G. Catalan, ${ }^{5,6}$ and A. Gruverman ${ }^{1}$
}

1. Department of Physics and Astronomy, University of Nebraska-Lincoln, Lincoln, NE 68588, USA

2. Department of Materials Science and Engineering, University of Wisconsin-Madison, Madison, WI 53706, USA

3. Department of Fluid Mechanics, Grupo Interdepartamental para la Colaboración Científica Aplicada (GRICCA), Universitat Politecnica de Catalunya, Barcelona, Spain

4. Department of Materials Science and Metallurgical Engineering, GRICCA, Universitat Politecnica de Catalunya, Barcelona, Spain

5. Institut Catala de Recerca i Estudis Avançats, (ICREA) Catalunya, Spain

6. Centre for Investigations in Nanoscience and Nanotechnology (CIN2), Consejo Superior de Investigaciones Cientificas (CSIC) and Institut Catala de Nanotecnologia (ICN), Campus de Bellaterra, Barcelona, Spain

Corresponding authors - G. Catalan \& A. Gruverman; gustau.catalan@cin2.es; agruverman2@unl.edu

\section{Abstract}

Ferroelectric materials are characterized by a permanent electric dipole that can be reversed through the application of an external voltage, but a strong intrinsic coupling between polarization and deformation also causes all ferroelectrics to be piezoelectric, leading to applications in sensors and high-displacement actuators. A less explored property is flexoelectricity, the coupling between polarization and a strain gradient. We demonstrate that the stress gradient generated by the tip of an atomic force microscope can mechanically switch the polarization in the nanoscale volume of a ferroelectric film. Pure mechanical force can therefore be used as a dynamic tool for polarization control and may enable applications in which memory bits are written mechanically and read electrically.

Ferroelectrics strongly couple changes in polarization of a material with its deformation, which gives rise to useful electromechanical phenomena including piezoelectric, electrostrictive, and flexoelectric effects. Coupling between polarization and homogeneous inplane strain can also be used to tune the symmetry and properties of ferroelectric thin films via strain engineering using different substrates $(1,2)$. For a ferroelectric, however, the spontaneous strain (unit-cell deformation) is identical whether the polarization is pointing up or down, so one does not immediately think of mechanical deformation as a viable mechanism to invert the polarization in a ferroelectric memory.

Stress can nevertheless be used to influence polarization. A large local deformation can be realized by pressing the sharp tip of an atomic force microscope (AFM) against the surface of a film, thereby causing a large stress concentration near the tip-sample contact. Previous studies showed suppression of the piezoelectric response under a sufficiently high loading force $(3,4)$, with almost complete recovery after stress release. Also, polarization vector rotation, caused by simultaneous application of an external electrical bias and tip-induced stress, has been observed in polycrystalline thin films $(4,5)$, although in these studies the random orientation of the polarization in the crystals allowed for partial ferroelastic rotation rather than pure $180^{\circ}$ inversion of polarization. The stress from adjacent grains also meant that the original polar configuration was recovered upon release of the tip pressure, so no permanent writing was achieved.

Recent reports by Lee et al. (6) show that the flexoelectric effect caused by strain gradients can create a strong imprint in uniaxial, perfectly oriented ferroelectric thin films, and flexoelectricity due to substrate bending was also invoked in the imprint of polarization in polycrystalline ferroelectric films (7). This suggests that strain gra- dients, rather than homogeneous strain, can be exploited for switching polarization and "writing" the domain bits into ferroelectric memories. This process is allowed by symmetry because a strain gradient, unlike a homogeneous strain, is an odd-parity tensor without inversion symmetry - strain gradients have directionality and polarity $(8,9)$. Although flexoelectricity is generally weaker than piezoelectricity, gradients grow in inverse proportion to the relaxation length, so very large flexoelectric effects can be achieved at the nanoscale (10-12).
A

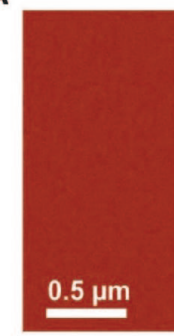

D

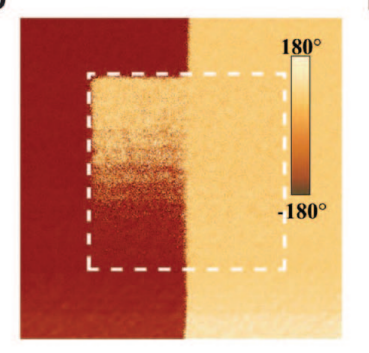

B

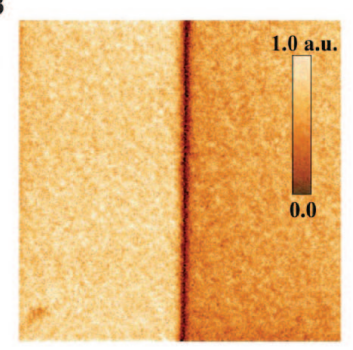

E

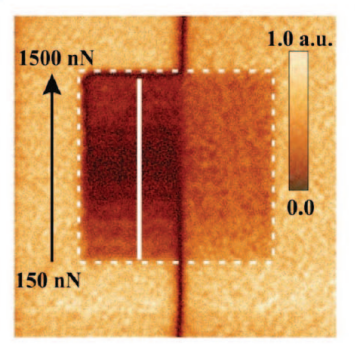

We have explored whether flexoelectricity can actively switch ferroelectric polarization by mechanically pushing AFM tips onto the surface of epitaxial single-crystalline $\mathrm{BaTiO}_{3}$ films, thereby inducing large and localized stresses. The films were fabricated by atomic layer controlled growth on atomically smooth (001) $\mathrm{Sr}$ $\mathrm{TiO}_{3}$ substrates with $\mathrm{La}_{0.67} \mathrm{Sr}_{0.33} \mathrm{MnO}_{3}$ conductive buffers that served as bottom electrodes $(13,14)$. Compressive stress induced by the substrate ensured that polarization was aligned in the direction perpendicular to the surface, and
Figure 1. Mechanically induced reversal of ferroelectric polarization. (A and B) PFM phase (A) and amplitude (B) images of the bidomain pattern electrically written in the $\mathrm{BaTiO}_{3}$ film. (C) Singlepoint PFM hysteresis loops of the $\mathrm{BaTiO}_{3}$ film. (D and E) PFM phase (D) and amplitude (E) images of the same area after the 1-by-1- $\mu \mathrm{m}^{2}$ area in the center (denoted by a dashed-line frame) has been scanned with the tip under an incrementally increasing loading force. The loading force was increasing in the bottom-up direction [denoted by a black arrow in (E)] from 150 to $1500 \mathrm{nN}$. (F) PFM amplitude as a function of the loading force obtained by cross-section analysis along the white vertical line in $(E)$.

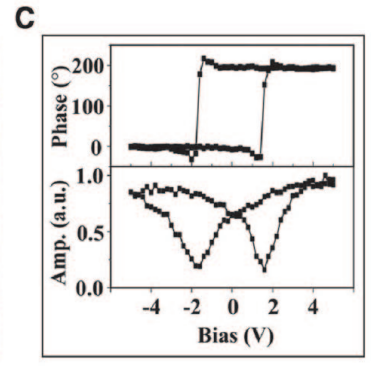

$\mathbf{F}$

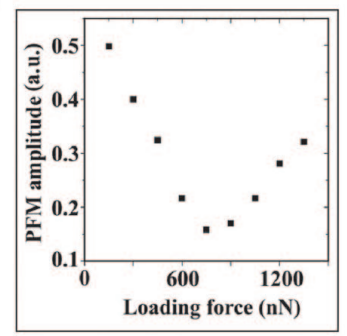


only $180^{\circ}$ inversion of polarization would be allowed. $\mathrm{BaTiO}_{3}$ films with a thickness of 12 unit cells, or $\sim 4.8 \mathrm{~nm}$, have been chosen so as to ensure epitaxial clamping and prevent mismatch strain relaxation (15).

Initial testing of the films by means of piezoresponse force microscopy (PFM) show that asgrown $\mathrm{BaTiO}_{3}$ films are in a single-domain state with out-of-plane polarization, indicating effective screening of the depolarizing field by surface adsorbates (16). Bipolar domain patterns can be generated conventionally with an electrically biased PFM tip: the film surface is scanned with a tip under $\pm 4-\mathrm{V}$ bias exceeding the coercive voltage. The 2-by-2- $\mu \mathrm{m}^{2}$ PFM images of this electrically written domain structure are shown in Figure 1, A and B. A typical value of the contact force during conventional PFM imaging is $\sim 30 \mathrm{nN}$. A stable and uniform PFM amplitude signal across the domain boundary illustrates effective electric switchability of the film and strong polarization retention. Local PFM spectroscopic measurements (Figure 1C) exhibit bipolar piezoelectric hysteresis loops.

The mechanical switching has been investigated by scanning a 1 -by-1- $\mu \mathrm{m}^{2}$ area of the bipolar domain pattern with the electrically grounded tip under an incrementally increasing loading force from 150 to $1500 \mathrm{nN}$, with a corresponding change in the applied stress from 0.5 to $5 \mathrm{GPa}$ (approximating the tip-surface contact area as a disk of $10 \mathrm{~nm}$ in radius). Note that, although the maximum local stress is very large, it is still well below the threshold $(\sim 20$ $\mathrm{GPa})$ for irreversible plastic damage of the Ba$\mathrm{TiO}_{3}$ surface (17). After that, a larger area of 2 by $2 \mu \mathrm{m}^{2}$ is imaged by conventional PFM with a low load of $30 \mathrm{nN}$ (Figure 1, D and E).

The tip-induced stress reverses the PFM phase contrast in the left half of the image in Figure 1D, from dark to bright, indicating inversion of the polarization from up to down. Figure 1E shows a nonmonotonous change in the corresponding PFM amplitude image of the flexoelectrically switched domain: Initially, the amplitude decreases as load increases and then, at an applied force of $\sim 750 \mathrm{nN}$, it increases again (Figure 1F). This type of behavior is analogous to the polarization-reversal process in conventional (voltage-induced) PFM (Figure S1) (18) in which the electromechanical amplitude signal passes through a minimum during switching, as in Figure 1C. This is caused by the formation of $180^{\circ}$ domains (antiparallel polarization) so that the net polarization and associated piezoelectric signal go through zero when the volume fractions of domains with opposite polarization become equal. Beyond that, the PFM amplitude grows again while the PFM phase is changed by $180^{\circ}$, indicating that the polarization has been inverted. For reference, the PFM images of conventionally (that is, electrically) switched domains in the same $\mathrm{BaTiO}_{3}$ film are shown in the supplementary materials (Figure S1) (18). The polarization patterns generated by an electrical bias (Figure S1) (18) and by mechanical load (Figure 1) are identical.

To rationalize the obtained results, we performed finite-element calculations of the strain

induced by the AFM tip when pressed against the $\mathrm{BaTiO}_{3}$ film surface (the structure is sketched in Figure 2A). The calculations are for a tip force of $1000 \mathrm{nN}$ over a tip-sample contact area of $10-\mathrm{nm}$ radius, and the calculated strain distributions for all the strain components are mapped (Figure S2) (18). Using the calculated strain gradients, the flexoelectric field is obtained by multiplying the strain gradient times the flexoelectric tensor and dividing by the dielectric constant $(9,19)$. To be conservative in our calculations, we use the theoretical values for the flexoelectric tensor coefficients (20), which are a thousand times smaller than the experimental ones $(21,22)$. The integral of the flexoelectric field is the flexoelectric potential, which we have calculated and is mapped in Figure 2B [details of the calculations are provided in (18)].

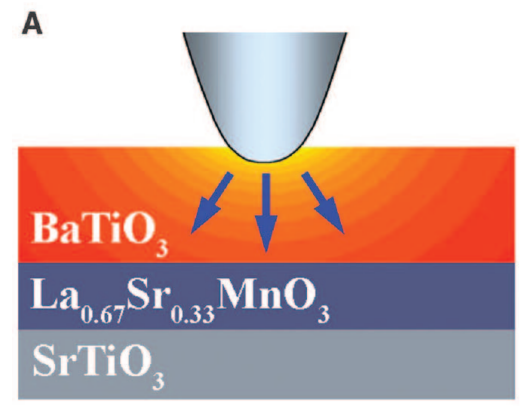

Figure 2. (A) Sketch of the strain gradient and associated flexoelectric field (arrows) induced by the AFM tip pushing on the surface of the $\mathrm{BaTiO}_{3} / \mathrm{La}_{0.67} \mathrm{Sr}_{0.33} \mathrm{MnO}_{3}$ heterostructure. (B) Variation of the flexoelectric voltage in the film region under the tip, assuming a compressive uniaxial stress of $3.2 \mathrm{GPa}$, equivalent to $1 \mu \mathrm{N}$ of force over a circular area of 10 $\mathrm{nm}$ in radius. The field is perpendicular to the voltage gradient and reaches a strength of $\sim 2$ $\mathrm{MV} / \mathrm{cm}$ near the surface. (C) Free-energy calculations for the epitaxially clamped $\mathrm{BaTiO}_{3}$ film without any tip pressure (blue curve), with homogeneous compressive stress of $3.2 \mathrm{GPa}$ (red curve), and with the calculated flexoelectricity from the tip-induced strain gradient (green curve). Flexoelectricity skews the double well, forcing polarization switching toward the stable downward state.

A

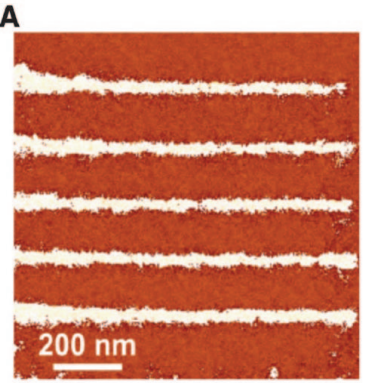

C

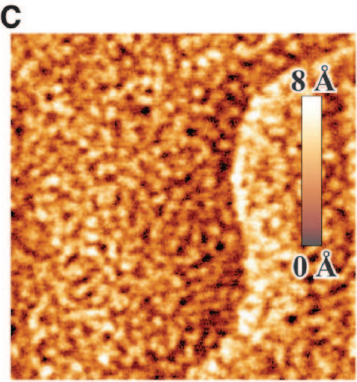

The flexoelectric field was also incorporated into free-energy calculations of the $\mathrm{Ba}$ $\mathrm{TiO}_{3}$ thin film epitaxially clamped on the $\mathrm{SrTiO}_{3}$ substrate (Figure 2C). Under a homogeneous compressive uniaxial stress (23), the height of the barrier separating the two energy minima is decreased, but the double well remains symmetric, and thus no specific polarity is favored, as expected from symmetry. Flexoelectricity, on the other hand, generates a polar bias consistent with the experimental observation of mechanical switching. When incorporated to the free energy, the flexoelectric bias destabilizes the positive side (upward-pointing polarization) of the double well and forces the switch to the downward-pointing polarization state, as observed. This is supported by PFM hysteresis loops measured

B

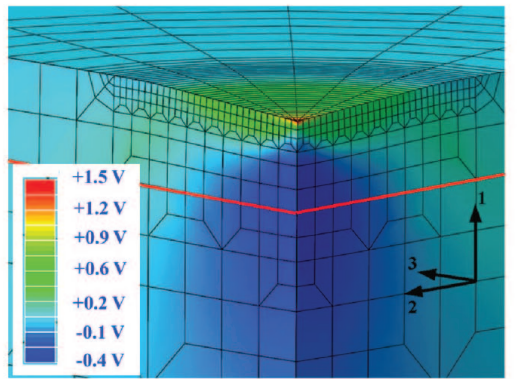

C

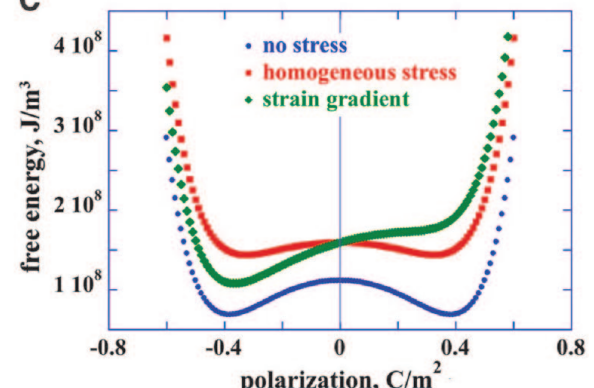

B

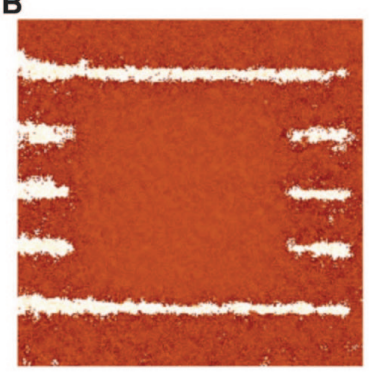

D

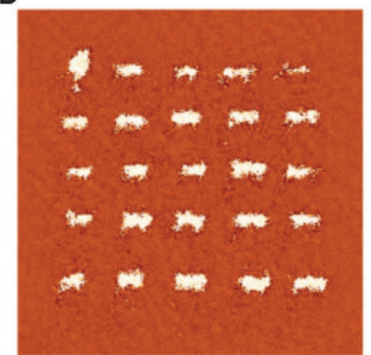

Figure 3. Fabrication of nanoscale domain patterns by mechanical means. (A) Domain lines mechanically written in the $\mathrm{BaTiO}_{3}$ film by scanning the film with a tip under a loading force of $1500 \mathrm{nN}$. (B) The same domain structure modified by electrical erasure of the mechanically written domains. Erasure has been performed by scanning the central segment with the tip under a dc $-3-\mathrm{V}$ bias. (C) Topographic image of the same area acquired after mechanical writing showing that the film surface was not affected by the writing process. (D) An array of flexoelectrically written dot domains illustrating the possibility of using mechanical writing for high-density data-storage application. 
during application of mechanical stress: As the tip pressure is increased, the positive coercive bias decreases, whereas the negative coercive bias remains intact (Figure S3) (18).

For the loading force of $1000 \mathrm{nN}$, the calculated flexoelectric field reaches a maximum of $2 \mathrm{MV} / \mathrm{cm}$, comparable with the intrinsic coercive field (theoretical) and that extracted from the piezoelectric hysteresis loops (experimental). Note that such a large flexoelectric field has been obtained in spite of our conservative choice of flexoelectric coefficients, supporting the feasibility of the flexoelectric switching mechanism. We stress that the Landau formalism provides an upper limit for the ideal (intrinsic) switching barrier; in practice, there will be defects that act as nucleation sites facilitating the switching at lower coercive fields. In real devices, therefore, the effective coercive field may be considerably lower than calculated here, meaning that the flexoelectric field will be capable of inducing switching at lower loads or larger thicknesses than assumed here.

There are several useful features of mechanical switching: (i) It generates stable domain patterns exhibiting no relaxation for days after switching, (ii) mechanically written domain patterns are electrically erasable, (iii) no damage to the sample surface caused by a high loading force was observed, and (iv) the mechanically written domains are nanoscopic. These features are illustrated in Figure 3. Mechanically written parallel linear domains, shown in Figure 3A, have been subsequently transformed into the pattern in Figure $3 \mathrm{~B}$ by electrically erasing central domain segments with a tip under a dc $-3-\mathrm{V}$ bias. The topographic image of the same area of the $\mathrm{BaTiO}_{3}$ film (Figure 3C), acquired after this procedure, does not exhibit any traces of surface deformation. Finally, Figure 3D shows an array of dot domains only $30 \mathrm{~nm}$ in size, written by abruptly alternating the tip load between 30 and $1500 \mathrm{nN}$ during scanning.

These results open up a way to write ferroelectric memory bits using mechanical force instead of electrical bias in data-storage devices. By converting mechanical stress into readable information, such devices would operate as a nanoscopic analog of typewriters that could be scaled up using a millipede-like scheme (24). The tip-sample contact area is typically less than 10-nm in radius, so switching can be highly localized, allowing fabrication of high-density domain patterns. Because no voltage is applied during mechanical switching, leakage and/or dielectric breakdown problems are minimized [in fact, even insulating tips can be used to mechanically write the domains, as shown in Figure S4 (18)]. Because electrodes are not required, the problems caused by their finite screening length (25) are also removed.

Conversely, if top electrodes were used, mechanical writing would enable the targeted poling of localized areas under the electrodes which is impossible using voltage, as the electric field is homogeneous in a parallel-plate capacitor. This suggests the possibility of controlled fabrication of domain walls underneath top electrodes, useful for electronic device applications employing physical properties of domain walls (26) that could be read in a nondestructive manner by PFM imaging (27) or by measuring the electroresistive effect (28).

\section{References and Notes}

1. K. J. Choi et al., Science 306, 1005 (2004).

2. G. Catalan et al., Phys. Rev. Lett. 96, 127602 (2006).

3. G. Zavala, J. H. Fendler, S. Trolier-McKinstry, J. Appl. Phys. 81, 7480 (1997).

4. A. Kholkin et al., Appl. Phys. Lett. 82, 2127 (2003).

5. A. Gruverman, A. Kholkin, A. Kingon, H. Tokumoto, Appl. Phys. Lett. 78, 2751 (2001).

6. D. Lee et al., Phys. Rev. Lett. 107, 057602 (2011).

7. A. Gruverman et al., Appl. Phys. Lett. 83, 728 (2003).

8. S. M. Kogan, Sov. Phys. Solid State 5, 2069 (1964).

9. J. F. Scott, J. Chem. Phys. 48, 874 (1968).

10. G. Catalan, L. J. Sinnamon, J. M. Gregg, J. Phys. Condens. Matter 16, 2253 (2004).

11. G. Catalan et al., Nat. Mater. 10, 963 (2011).

12. M. S. Majdoub, P. Sharma, T. Çağin, Phys. Rev. $B 78,121407$ (2008).

13. C. B. Eom et al., Science 258, 1766 (1992).

14. L. J. Belenky, X. Ke, M. Rzchowski, C. B. Eom, J. Appl. Phys. 97, 10J107 (2005).

15. In our studies, the flexoelectric switching has been demonstrated in films with thickness of up to 48 unit cells.

16. D. D. Fong et al., Phys. Rev. Lett. 96, 127601 (2006).
17. Y. Gaillard, A. Hurtado Macías, J. MuñozSaldaña, M. Anglada, G. Trápaga, J. Phys. D Appl. Phys. 42, 085502 (2009).

18. Supplementary materials follow the Notes section.

19. W. Ma, Phys. Status Solidi B 245, 761 (2008).

20. R. Maranganti, P. Sharma, Phys. Rev. B 80, 054109 (2009).

21. W. Ma, L. E. Cross, Appl. Phys. Lett. 88, 232902 (2006).

22. J. Hong, G. Catalan, J. F. Scott, E. Artacho, J. Phys. Condens. Matter 22, 112201 (2010).

23. A. Yu. Emelyanov, N. A. Pertsev, A. L. Kholkin, Phys. Rev. B 66, 214108 (2002).

24. P. Vettiger et al., IEEE Trans. NanoTechnol. 1, 39 (2002).

25. V. Nagarajan et al., J. Appl. Phys. 100, 051609 (2006).

26. G. Catalan, J. Seidel, R. Ramesh, J. F. Scott, Rev. Mod. Phys. 84, 119 (2012).

27. D. A. Bonnell, S. V. Kalinin, A. Kholkin, A. Gruverman, MRS Bull. 34, 648 (2009).

28. V. Garcia et al., Nature 460, 81 (2009).

Acknowledgments - A.G and G.C. conceived the idea, designed the experiment, and wrote the paper; H.L. implemented experimental measurements; C.-W.B. fabricated the samples; C.-B.E. supervised sample preparation and reviewed the paper; and D.E.O., J.A., and G.C. performed finite-element and free-energy calculations. G.C. and A.G. thank the Leverhulme Trust for international network funding ( $F / 00203 / V$ ) for the funds that have enabled this collaboration. G.C. acknowledges financial support from grants MAT2010-10067-E and MAT2010-17771, and J.A. acknowledges support from grant MAT2011-23375 (Ministerio de Educación, Ciencia e Innovación). The work at University of Nebraska-Lincoln was supported by the Materials Research Science and Engineering Center (NSF grant DMR-0820521) and by the U.S. Department of Energy (DOE), Office of Basic Energy Sciences, Division of Materials Sciences and Engineering (DOE grant DE-S(0004876). The work at Univ. of Wisconsin-Madison was supported by the NSF under grant ECCS-0708759.

Supplementary Materials, including Materials and Methods, Supplementary Text, Figures $\mathrm{S}_{1}$ to $\mathrm{S}_{4}$, and References, follow 


\section{Supplementary Materials for}

\section{Mechanical Writing of Ferroelectric Polarization}

H. Lu, C.-W. Bark, D. Esque de los Ojos, J. Alcala, C. B. Eom, G. Catalan,* A.

Gruverman $^{1 *}$

*To whom correspondence should be addressed. E-mail: gustau.catalan@cin2.es (G.C.); agruverman2@unl.edu (A.G.)

Published 6 April 2012, Science 336, 59 (2012)

DOI: 10.1126/science.1218693

This PDF file includes:

Materials and Methods

Supplementary Text

Figs. S1 to S4

References 


\section{Materials and Methods}

Single-crystalline epitaxial $\mathrm{BaTiO}_{3}$ heterostructures have been grown by pulsed laser deposition (PLD) on atomically smooth (001) $\mathrm{SrTiO}_{3}$ substrates with 30 -nm-thick $\mathrm{La}_{0.67} \mathrm{Sr}_{0.33} \mathrm{MnO}_{3}$ electrode. Reflection high-energy electron diffraction (RHEED) has been used for in-situ monitoring of the layer-by-layer growth process. Before deposition, low angle miscut $\left(<0.1^{\circ}\right) \mathrm{SrTiO}_{3}$ substrates were etched using buffered HF acid for 90 seconds to maintain Ti-termination and then were annealed in oxygen at $1000^{\circ} \mathrm{C}$ for 12 hours to create atomically smooth surfaces with single-unit-cell-height steps. During deposition of all the layers substrate temperature was maintained at $680{ }^{\circ} \mathrm{C}$ with chamber oxygen pressure kept at 150 mTorr. The samples were annealed at growth temperature and 1 atm oxygen pressure for 30 minutes and then cooled down to room temperature.

Polarization imaging and local switching spectroscopy has been performed using a resonant-enhanced Piezoresponse Force Microscopy (MFP-3D, Asylum Research). Conductive silicon cantilevers (PPP-EFM, Nanosensors) have been used in this study. PFM hysteresis loops were obtained at fixed locations on the $\mathrm{BaTiO}_{3}$ electrodes as a function of switching pulse amplitude (pulse duration was $25 \mathrm{~ms}$ ) superimposed on ac modulation bias with amplitude of $0.8 \mathrm{~V}_{\mathrm{p}-\mathrm{p}}$ at $320 \mathrm{kHz}$. Tip contact forces have been calibrated by measuring force-distance curves.

The finite element simulations were performed for an AFM tip in contact with a $\mathrm{BaTiO}_{3}$ film of $5 \mathrm{~nm}$ thickness attached to a semi-infinite $\mathrm{SrTiO}_{3}$ substrate. The full anisotropy of the contact behavior was taken into account through the following coefficients for the stiffness matrices: (1) for $\mathrm{BaTiO}_{3}, \mathrm{C}_{11}=358.1 \mathrm{GPa}, \mathrm{C}_{12}=115.2 \mathrm{GPa}$ and $\mathrm{C}_{14}=149.8 \mathrm{GPa}$; (2) for the $\mathrm{SrTiO}_{3}$ substrate, $\mathrm{C}_{11}=421 \mathrm{GPa}, \mathrm{C}_{12}=122.1 \mathrm{GPa}, \mathrm{C}_{14}=$ 
133.2 GPa [i]. As compared to an ideal spherical shape, a blunt punch configuration better reproduces the nanoscale surface irregularities present in a real AFM tip.

\section{Supplementary Text}

\section{Piezoresponse signal variation due to electrically-induced polarization reversal}

Figure S1 shows conventional PFM images of the $\mathrm{BaTiO}_{3}$ film where an area in the center has been scanned with a tip under an incrementally increasing electrical dc bias before PFM imaging. The PFM amplitude contrast in Fig S1(A) gradually changes with the applied electric dc bias. Specifically, initially the PFM amplitude signal decreases and then, after reaching a minimum value at the applied bias of $1.25 \mathrm{~V}$, it starts to increase (Fig S1(C)). This change in PFM amplitude is analogous to what was observed in the measurements as a function of mechanical stress (Figs $1 \mathrm{D}$ and $1 \mathrm{~F}$ of the main text). A corresponding change in the PFM phase image (Fig S1(B)) shows contrast inversion at the same dc bias at which the PFM amplitude reaches its minimum, also in complete analogy with the mechanically induced phase change in Fig 1E. In both cases, the PFM response can be interpreted in terms of nucleation and growth of $180^{\circ}$ domains with polarization antiparallel to the initial state, whose volume fraction grows under increasing dc bias. A minimum in the PFM amplitude corresponds to the situation at which there are equal fractions of upward and downward polarization, so that the piezoelectric responses cancel each-other.

The results show that there is no fundamental difference in the switching response induced by conventional electric biasing (Fig S1) and that induced by mechanical loading (Figs 1D and 1E of the main text), indicating that flexoelectricity is an effective substitute for voltage in ferroelectric switching. 


\section{Flexoelectric calculations}

The flexoelectric field is given by [ii, iii]:

$$
E_{h}=\frac{f_{i j k l}}{\varepsilon_{i h}} \frac{\partial e_{k l}}{\partial x_{j}}
$$

where $f_{i j k l}$ is the flexoelectric tensor, $\varepsilon_{i h}$ the dielectric constant, $e_{j \mathrm{k}}$ the strain and $x_{l}$ are the spatial coordinates of the film. The vertical component of the tip-induced flexoelectric field is therefore:

$$
E_{3}=\frac{f_{3311}}{\varepsilon_{33}} \frac{\partial e_{11}}{\partial x_{3}}+\frac{f_{3322}}{\varepsilon_{33}} \frac{\partial e_{22}}{\partial x_{3}}+\frac{f_{1313}}{\varepsilon_{33}} \frac{\partial e_{13}}{\partial x_{1}}+\frac{f_{2323}}{\varepsilon_{33}} \frac{\partial e_{23}}{\partial x_{2}}+\frac{f_{3333}}{\varepsilon_{33}} \frac{\partial e_{33}}{\partial x_{3}}
$$

The dielectric constant is $\varepsilon_{3}=\varepsilon_{0} \varepsilon_{r}$, where $\varepsilon_{0}$ is the permittivity of vacuum and $\varepsilon_{r}=30$ for compressively strained $\mathrm{BaTiO}_{3}$ on $\mathrm{SrTiO}_{3}$. Though the shear flexoelectric coefficients $\left(f_{1313}\right.$ and $\left.f_{2323}\right)$ are comparable to the transverse ones ( $f_{3311}$ and $\left.f_{3322}\right)$ [iv], the finite element calculations (Fig S2) show that the shear strain gradients are an order of magnitude smaller than the transverse ones, so we can neglect the shear terms. Conversely, though the longitudinal flexoelectric coefficient $\left(f_{3333}\right)$ is an order of magnitude smaller than the others [iv], the longitudinal deformation is an order of magnitude larger than the transverse one, so it cannot be neglected. Thus, the flexoelectric field under the tip is:

$$
E_{3}=\frac{f_{3311}}{\varepsilon_{33}} \frac{\partial e_{11}}{\partial x_{3}}+\frac{f_{3322}}{\varepsilon_{33}} \frac{\partial e_{22}}{\partial x_{3}}+\frac{f_{3333}}{\varepsilon_{33}} \frac{\partial e_{33}}{\partial x_{3}}
$$

The strain fields $e_{11}, e_{22}$ and $e_{33}$ have been calculated by finite element methods using parameters listed below in the Materials and Methods section. Once the flexoelectric field $E_{3}$ is known, its effect is calculated by adding the term $-E_{3} P$ to the standard LandauDevonshire free energy [v] of an epitaxial film under uniaxial compression. 
To visualize the results and facilitate comparison with experiment, it is useful to introduce a concept of a "flexoelectric potential", V, whose gradient is the flexoelectric field: $E_{3} \equiv \frac{\partial V}{\partial x_{3}}$. Comparing this with Eq. 3, we obtain the flexoelectric potential as:

$$
V=\frac{f_{3311}}{\varepsilon_{33}} e_{11}+\frac{f_{3322}}{\varepsilon_{33}} e_{22}+\frac{f_{3333}}{\varepsilon_{33}} e_{33}
$$

The flexoelectric potential under the tip, measured in Volts, is shown in Figure 2b of the article. Note that though Eq. 4 would imply that a homogeneous deformation also causes a non-zero voltage, this would however be constant across the film and would therefore have no physical consequence. Only potential differences matter, i.e. there is only a field if there is a potential gradient, which is the case when the induced deformation ininhomogeneous.

\section{Stress and piezoelectricity}

The mechanical boundary conditions are asymmetric in our film, as there is a large stress concentration at the top surface and virtually no stress at the bottom surface. Standard piezoelectric coupling will therefore lead to a polarization gradient directly proportional to the strain gradient, and therefore to a depolarization field that, if sufficiently large and unscreened may conceivably be able to cause switching. However, this mechanism cannot be invoked when the local depolarizing field is compensated, as is the case in our experiments, where we have used grounded metallic tips and/or metallic electrodes. More generally, piezoelectric switching can also be discarded because the piezoelectric effect should lead to the back-switching of domains of both polarization directions, up and down, whereas our experimental data show the switching only of the 
domains oriented up but not the opposite. This is incompatible with the piezoelectric mechanism and fully consistent with the flexoelectric mechanism.

Another, more relevant effect of compressive vertical stress is related to decrease of tetragonality of the film and thus its coercivity. This can be seen in Fig 2C of the main article, showing that the energy barrier between the two potential wells is lower when the film is under uniaxial compression. This lower barrier means that smaller biases are sufficient to switch it. Figure S3 shows several PFM hysteresis loops acquired in the $\mathrm{BaTiO}_{3}$ film at different tip-applied loading forces. It can be seen that, under increasing load, the hysteresis loops become both narrower, signaling reduced coercivity, and increasingly asymmetric, due to the flexoelectric bias. We can quantify this:

(1) We define the coercivity as the half-width of the hysteresis loop, measured as the difference between positive and negative coercive voltages divided by two: $\mathrm{V}_{\mathrm{c}}=\left(\mathrm{V}_{\mathrm{c}}{ }^{+}-\right.$ $\mathrm{V}_{\mathrm{c}}{ }^{-}$)/2. The coercivities for the films under increasing vertical force of $50 \mathrm{nN}, 250 \mathrm{nN}$, $500 \mathrm{nN}$ and $1000 \mathrm{nN}$ are, respectively, $1.6 \mathrm{~V}, 1.3 \mathrm{~V}, 1.0 \mathrm{~V}, 0.6 \mathrm{~V}$. This monotonical decrease in coercivity reflects the decrease in the average tetragonality of the film due to the vertical compression.

(2) We define the offset bias as the shift of the centre of the hysteresis loop, which is half the sum of positive and negative coercive voltages: $V_{o f f}=\left(V_{c}{ }^{+}+V_{c}{ }^{-}\right) / 2$. The offset for the films under increasing force is $0 \mathrm{~V},-0.1 \mathrm{~V},-0.4 \mathrm{~V},-0.7 \mathrm{~V}$. There is a monotonical shift of the hysteresis loops towards negative values as the pressure on the tip is increased, consistent with an increasing flexoelectric bias.

Note also that the positive coercive field is reduced while the negative coercive field seemingly does not. This is a direct consequence of the simultaneous narrowing and biasing of the loops. As the hysteresis loops get narrower, both the negative and positive 
coercive fields move towards zero. For the negative coercive field, the negative shift caused by the bias is compensated by the positive shift caused by the reduction in bias, with the net effect being that the negative coercive field barely moves. Meanwhile, for the positive coercive field, the negative bias and reduction in coercivity both act in the same direction and thus the positive side of the loop rapidly shifts left. At a sufficiently high load ( $>1000 \mathrm{nN})$, the positive coercive field is shifted to the negative voltage range, indicating that without external voltage only the downward polarization state is stable.

\section{Mechanical switching with an insulating tip}

Figure S4 illustrates that mechanical switching is possible even with an insulating tip (PFM readout of course still requires a conducting tip). Mechanical switching was performed with a non-conductive silicon tip by applying a force of $1400 \mathrm{nN}$ during scanning. The resulting polarization pattern was then visualized in a conventional PFM mode using a metal-coated tip. These results demonstrate that the mechanical switching is impervious to electrical boundary conditions (we have now done it both with grounded conducting tips and with insulating tips). The demonstration of switching with mechanically tougher non-metallic tips (Si or diamond) that are not prone to mechanical wear and accompanying electrical degradation as metal-coated tips is helpful in the implementation in more robust devices. 
A

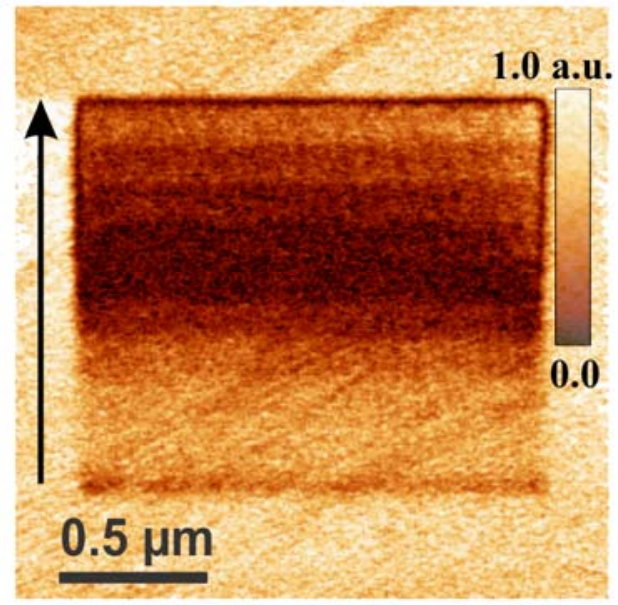

B

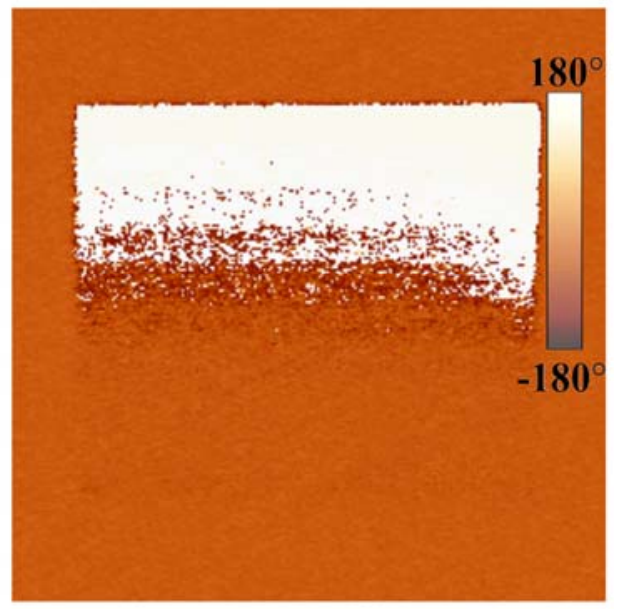

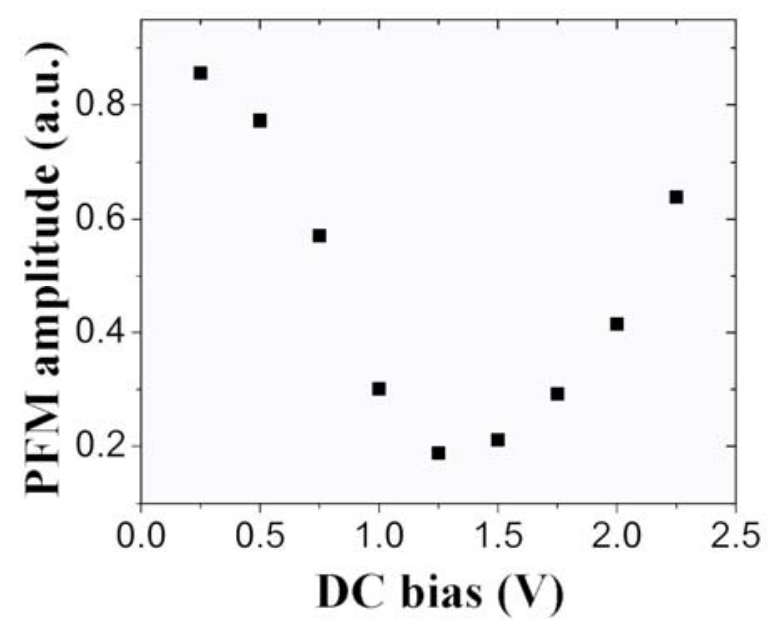

Figure S1. Electrically-induced reversal of ferroelectric polarization in the $\mathrm{BaTiO}_{3}$ film. (A) PFM amplitude and (B) phase images of the film acquired after the central area has been scanned with the tip under an incrementally increasing dc bias (image size is $3 \times 3 \mathrm{~m}^{2}$ ). The $\mathrm{dc}$ bias was increasing in the bottom-up direction (marked by a black arrow in (A)) from $0 \mathrm{~V}$ to $2.3 \mathrm{~V}$. (C) PFM amplitude as a function of a dc bias obtained by cross-section analysis of the poled area in (A). 
A

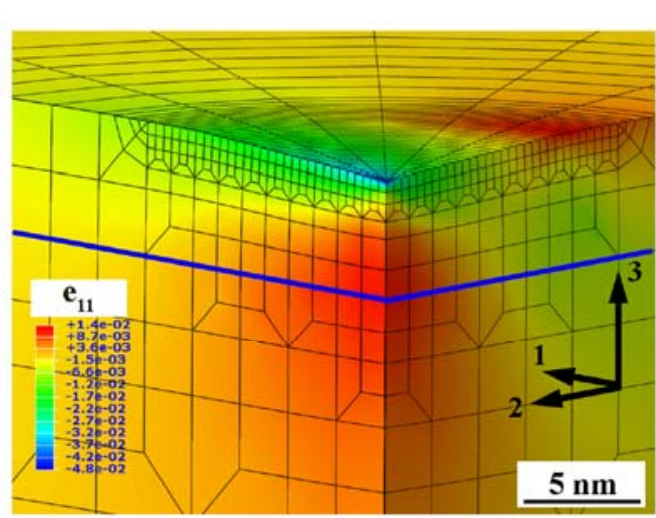

C

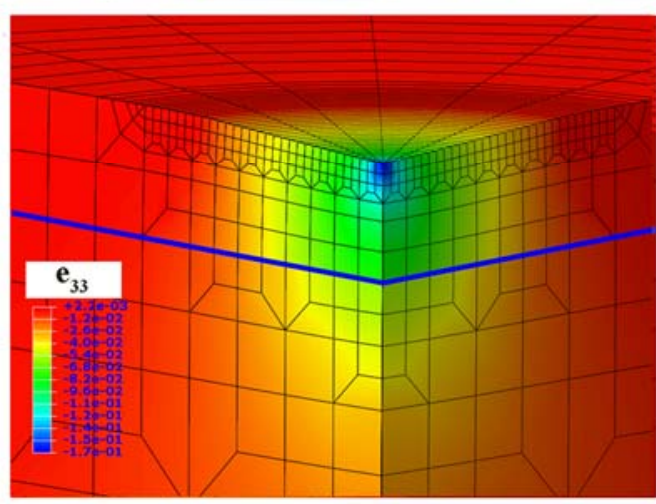

B

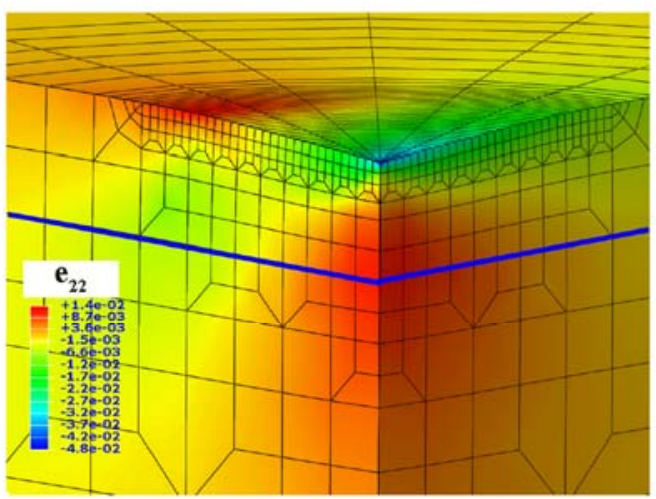

D

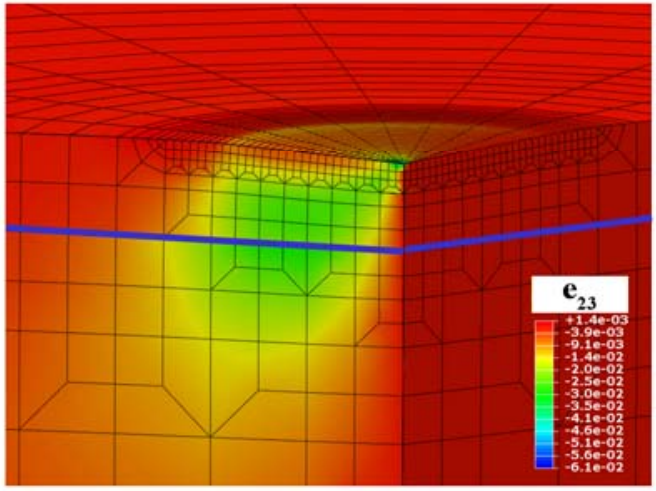

Figure S2. Finite element calculations of the deformations fields along the (A) $x$ $\left(e_{11}\right),(\mathrm{B}) y\left(e_{22}\right)$, and $(\mathrm{C}) \mathrm{z}\left(e_{33}\right)$ directions under pressure from the AFM tip. (D) Finite element calculation map of the shear strain $\left(e_{13}=e_{23}\right)$. All the strain components show gradients, with the shear strain gradient being the smallest one. 
A
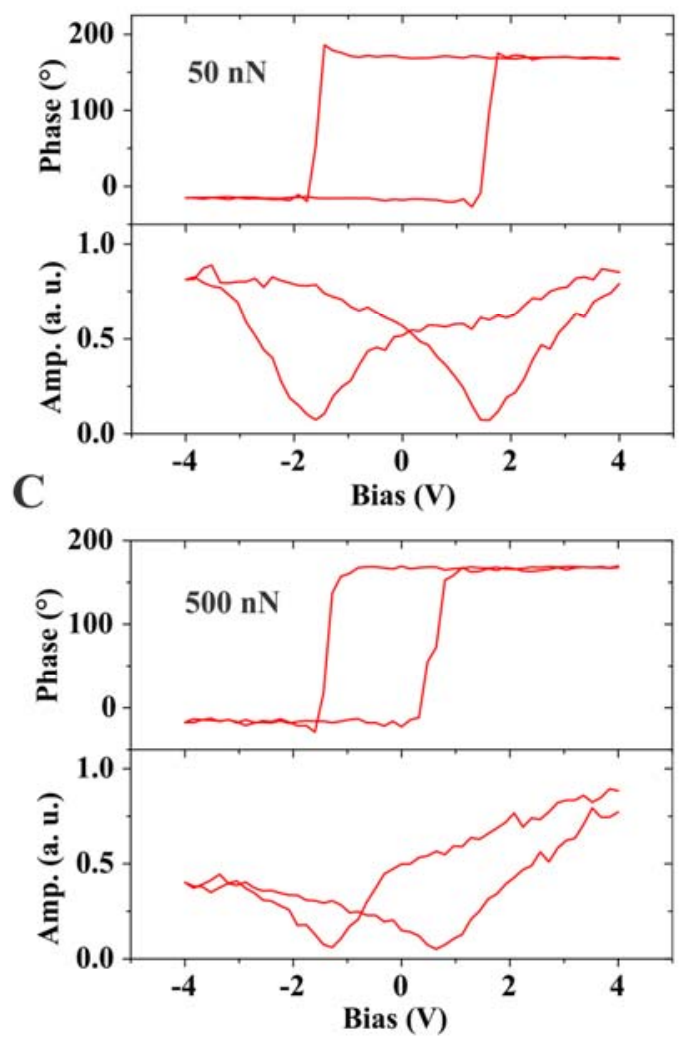

B
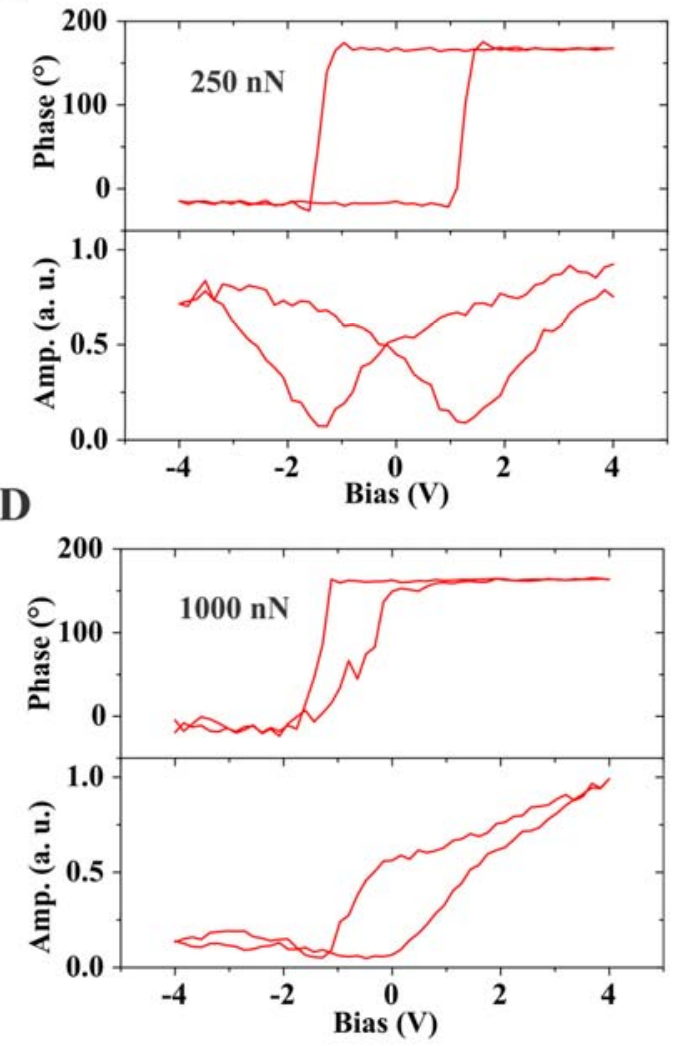

Figure S3. PFM hysteresis loops acquired at different loading forces. Note a shift of the loops towards the negative bias upon the load increase. 

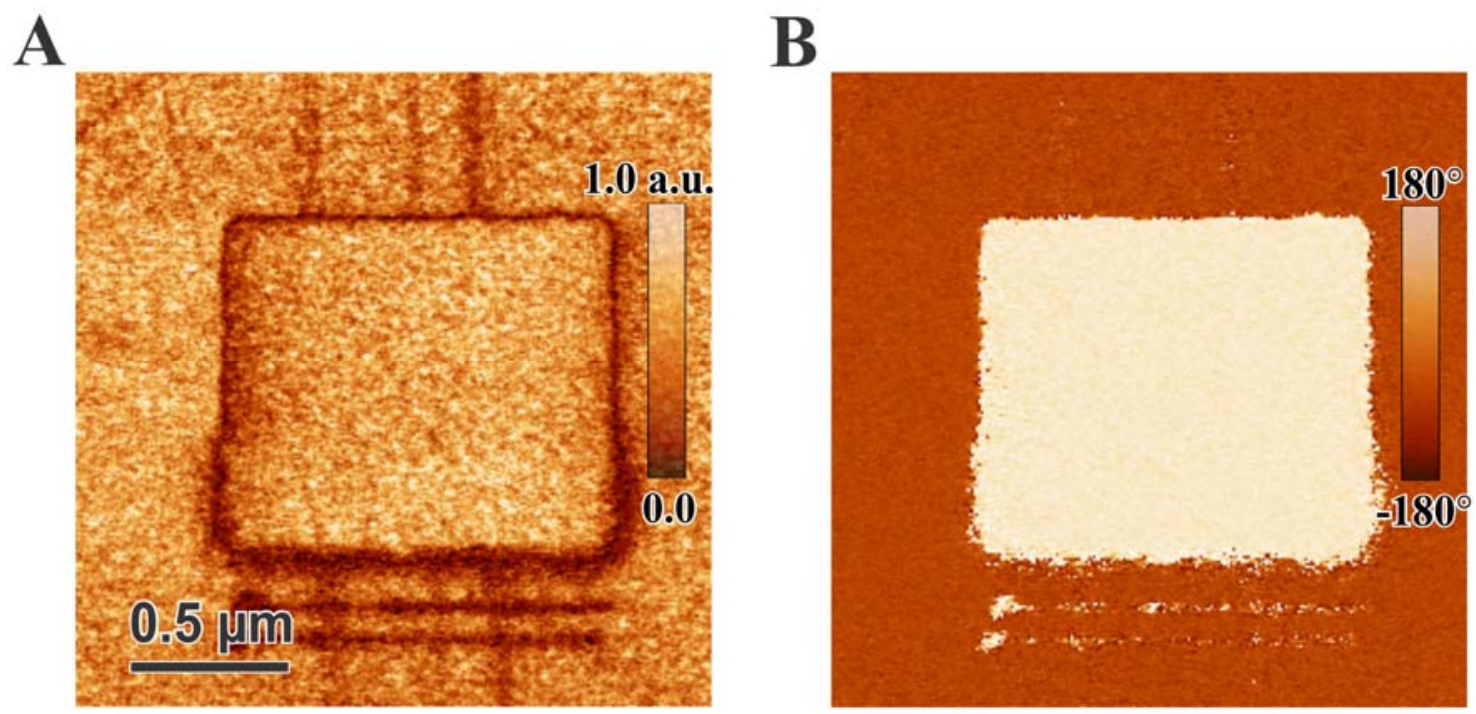

Figure S4. PFM amplitude (A) and phase (B) images of the domain pattern generated by mechanical switching using a non-conductive Si tip under a $1400 \mathrm{nN}$ load and visualized in a conventional PFM mode using a PtIr-coated Si tip.

\footnotetext{
${ }^{\text {i }}$ Piskunov, S., Heifets, E., Eglitis, R.I., Borstel, Bulk properties and electronic structure of $\mathrm{SrTiO}_{3}, \mathrm{BaTiO}_{3}, \mathrm{PbTiO}_{3}$ perovskites: an ab inition HF/DFT study. Comp. Mater. Sci. 29, 165 (2004).

${ }^{\text {ii }}$ Ma, W. A study of flexoelectric coupling associated internal electric field and stress in thin film ferroelectrics. Phys. Stat. Sol. (b) 245, 761-768 (2008).

iii Lee, D. et al. Giant Flexoelectricity Effect in Ferroelectric Epitaxial Thin Films. Phys. Rev. Lett. 107, 057602 (2011).
} 
iv Maranganti, R. and Sharma, P. Atomistic determination of flexoelectric properties of crystalline dielectrics. Phys. Rev. B 80, 054109 (2009).

${ }^{v}$ Yu. Emelyanov, A., Pertsev, N. A., and Kholkin, A. L., Effect of external stress on ferroelectricity in epitaxial thin films, Phys. Rev. B 66, 214108 (2002). 